\title{
Study and Analysis of Relay Structure for Wireless Power Transmission System via Electromagnetic Resonant Coupling
}

\author{
WANG Hao ${ }^{1, a^{*}}$, WANG An-na ${ }^{2, b}$, ZHAO Qiang ${ }^{3 . c}$ O Hyon Gyu ${ }^{4, d}$ \\ ${ }^{1}$ College of Information Science and Engineering, Northeastern University, China. \\ ${ }^{2}$ College of Information Science and Engineering, Northeastern University, China. \\ ${ }^{3}$ College of Information Science and Engineering, Northeastern University, China. \\ ${ }^{4}$ College of Information Science and Engineering, Northeastern University, China.
}

awanghao7405@163.com, bwanganna@mail.neu.edu.cn, 'Inshzq@126.com , dohk1980@126.com

Keywords: relay structure; couple mode; energy exchange; load voltage ratio; relay coefficient

Abstract :In order to analysis the transmission efficiency of wireless power transmission system via electromagnetic resonant coupling with relay structure, first we build the system model, analyze the relay system power model with couple mode theory, derive the same power change rule compare with non-relay system. Take advantage of electromagnetic simulation software to make an system analysis with the magnetic field distribution of the power transmission system, then analysis the circuit model of relay system to get the load power ratio, we propose the motion as relay coefficient. In this way, we derive the relationship between the load power ratio and relay coefficient. According to equivalent relation of load power, we make a mode of relay coefficient which could calculate reasonable relay coefficient. Finally, we verify the accuracy of this mode.

\section{Introduction}

Wireless power transmission is an ideal power transmission way which we are pursuing, this power transmission method could get rid of the constraint of the physical medium, therefore , wireless power transmission is also called contactless power transmission. An energy transmission channel which is built by electric field and magnetic field is used for power transmission. This technology is a multidisciplinary complex crossover technology, which conclude electromagnetic field theory, power electronic technology, radio-frequency technique, circuit theory and coupled-mode theory and so on. This technology could make much acceleration of our social production and life[1-3].

Wireless power transfer technology could be divided into three kinds: the first kind is electromagnetic inductive coupling, take advantage of electromagnetic induction principle, use loosely coupled transformer to realize power wireless transfer. This technology has advantages such as flexible and convenient and good reliability. University of Auckland did research on this technology earliest, and they also did a completion theory and repertoire, which is already put into production in commission. The second kind is microwave wireless power transmission technology. Power could be transfer from transmitting antenna to receiving antenna by high-frequency electromagnet wave. The third kind is the electromagnetic coupling resonance technology, it also called witricity, this technology was first proposed and studied by MIT team. As a new power transmission technology[4-7], the electromagnetic coupling resonance technology is based on strong coupling 


\section{Electrical model}

Common WPT system was consist of two sub-resonance circuits, relay structure WPT system has three sub-resonance circuits, and the three sub-resonance circuits have the same resonant parameters, the equivalent circuit model shown as figure 1 . Because the inherent loss impedance of relay structure is so little that the circuit has a great quality factor[8].

In figure 1 , the $R_{s}$ is internal resistance of high-frequency electric source; $R_{L}$ is load impedance; $R_{s o}$ is the internal resistance of transmitting terminal; $L_{s}$ and $C_{s}$ are the equivalent inductance and equivalent capacitance of transmitting terminal; $R_{R E P}$ is the internal resistance of relay structure; $L_{R E P}$ and $C_{R E P}$ are the equivalent inductance and equivalent capacitance of relay structure; $R_{r o}$ is the internal resistance of receiving terminal; $L_{r}$ and $C_{r}$ are the equivalent inductance and equivalent capacitance of receiving terminal; $k_{1}$ and $k_{2}$ are the coupling factors of the transmitting terminal between relay structure and receiving terminal between relay structure.

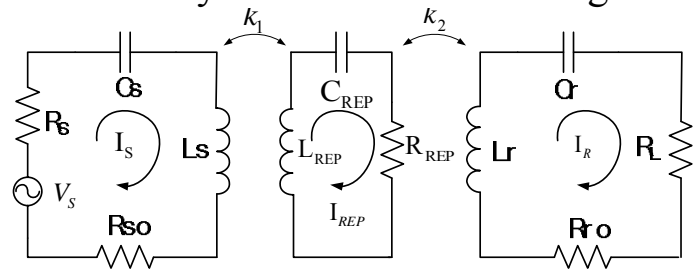

Fig.1 Equivalent circuit model

Make equivalent analysis of system circuit configuration with Kirchhoff Voltage Law, it got the equation of transmission system:

$$
\left\{\begin{array}{l}
\left(1-\frac{\omega_{0}^{2}}{\omega^{2}}\right) I_{S}+k_{1} I_{R E P} \sqrt{\frac{L_{R E P}}{L_{S}}}=0 \\
k_{1} I_{S} \sqrt{\frac{L_{S}}{L_{R E P}}}+\left(1-\frac{\omega_{0}^{2}}{\omega^{2}}\right) I_{R E P}+k_{2} I_{R} \sqrt{\frac{L_{R}}{L_{R E P}}}=0 \\
\left(1-\frac{\omega_{0}^{2}}{\omega^{2}}\right) I_{R}+k_{2} I_{R E P} \sqrt{\frac{L_{R E P}}{L_{S}}}=0
\end{array}\right.
$$

It could deduce the load voltage through the formal (1);

$$
\begin{aligned}
& V_{L}=I_{R} R_{L}=\frac{V_{S} \omega_{0} \sqrt{L_{S} L_{R}} / R_{1} R_{2}}{\frac{k_{1}}{k_{2}} Q_{1}+\frac{k_{2}}{k_{1}} Q_{2}+\frac{1}{Q_{R E P}} \frac{1}{k_{1} k_{2}}} \\
& \frac{V_{L}}{V_{S}}=\frac{1}{\frac{k_{1}}{k_{2}}+\frac{k_{2}}{k_{1}}}
\end{aligned}
$$

$V_{L}$ is load voltage, because the transmitting terminal, receiving terminal and relay structure have the same structural characteristics and resonant parameter, so, And the transmitting terminal has the same quality factor with receiving terminal, The quality factor of relay structure is so great, The radiation impedance of transmitting terminal is equal to the radiation impedance of receiving terminal, and they are much less than the load impedance and internal resistance of high-frequency electric source, they could be ignored, so the formal (2) could be simplify as formal (3).

When $k_{1}=k_{2}, V_{L}$ gets the maximum value, the relay structure is located in the middle of axis between transmitting terminal and receiving terminal at this time, load of system could get the maximum power. Shown as figure 2, the transmitting terminal and receiving terminal are fixed, the relay structure moves along the axis, so the WPT system is divide into two ports, as the relay structure changed the transmitting characteristic is also changed. 
The coupling coefficient between transmitting terminal and relay structure could be expressed by the mutual inductance and self-inductance, shown as formal (4), the coupling coefficient between receiving terminal and relay structure also could be expressed by the mutual inductance and self-inductance, shown as formal (5).

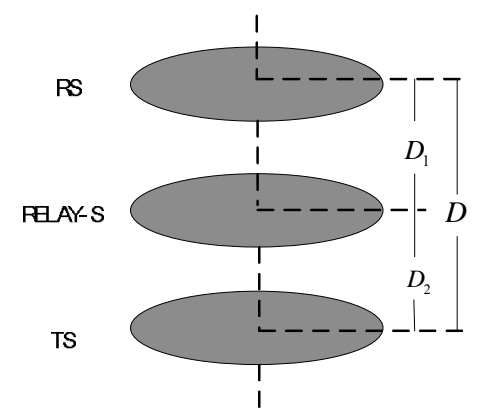

Fig.2 Diagram of relay antenna position

$$
\begin{aligned}
& k_{1}=\frac{M_{1}}{\sqrt{L_{S} L_{R E P}}} \\
& k_{2}=\frac{M_{2}}{\sqrt{L_{R} L_{R E P}}} \\
& M_{1}=\frac{\pi \mu_{0} n r^{4}}{2 D_{1}^{3}}=\frac{\Delta}{D_{1}^{3}} \\
& M_{2}=\frac{\pi \mu_{0} n r^{4}}{2 D_{2}{ }^{3}}=\frac{\Delta}{D_{2}{ }^{3}}
\end{aligned}
$$

$\Delta=\frac{\pi \mu_{0} n r^{4}}{2}$ is constant value for fixed system.

$$
\begin{aligned}
& D=\alpha D_{1} \\
& D_{2}=D-D_{1}=(\alpha-1) D_{1}
\end{aligned}
$$

$\alpha$ is relay coefficient, it is the specific value between the total transmission distance and the primary transmission distance; $n$ is number of windings; $r$ is radius of antenna; $D$ is the vertical dimension between transmission terminal and receiving terminal; because the transmission terminal, relay structure and receiving terminal have the same structural properties, so the $\Delta=\pi \mu_{0} n r^{4} / 2$ is a constant value, and $L_{S}=L_{R}=L_{R E P}=L$.

$$
\begin{aligned}
& k_{1}=\frac{M_{1}}{\sqrt{L}}=\frac{\frac{\Delta}{D_{1}^{3}}}{\sqrt{L}} \\
& k_{2}=\frac{M_{2}}{\sqrt{L}}=\frac{\frac{\Delta}{D_{2}^{3}}}{\sqrt{L}}=\frac{\frac{\Delta}{(\alpha-1)^{3} D_{1}^{3}}}{\sqrt{L}} \\
& \frac{V_{L}}{V_{S}}=\frac{1}{\frac{k_{1}}{k_{2}}+\frac{k_{2}}{k_{1}}}=\frac{1}{(\alpha-1)^{3}+\frac{1}{(\alpha-1)^{3}}}
\end{aligned}
$$

The relay coefficient change as the load voltage change, the corresponding change rule shown in figure 3 , when $\alpha=2$, it get the maximum of load voltage, with the relay coefficient increase or decrease, the load voltage decrease quickly, so it affect the transfer efficiency for total WPT system. When the relay structure is so closed to the transmission terminal or receiving terminal, the transfer efficiency of the WPT system would be so low.

Compare with the common WPT system, the relay structure WPT system could get a better 
load voltage ratio, according to formal (10), there must be a maximum load power point, so it could be analyzed that there must be a vertical position range for relay structure to get a load voltage ratio which is not under the common WPT system, take advantage of the equivalent power method to get a equivalent relay coefficient range, it could acquire a reasonable range of relay structure though this range. First to build the circuit model, and then calculate the load voltage ratio of common WPT system by KVL.

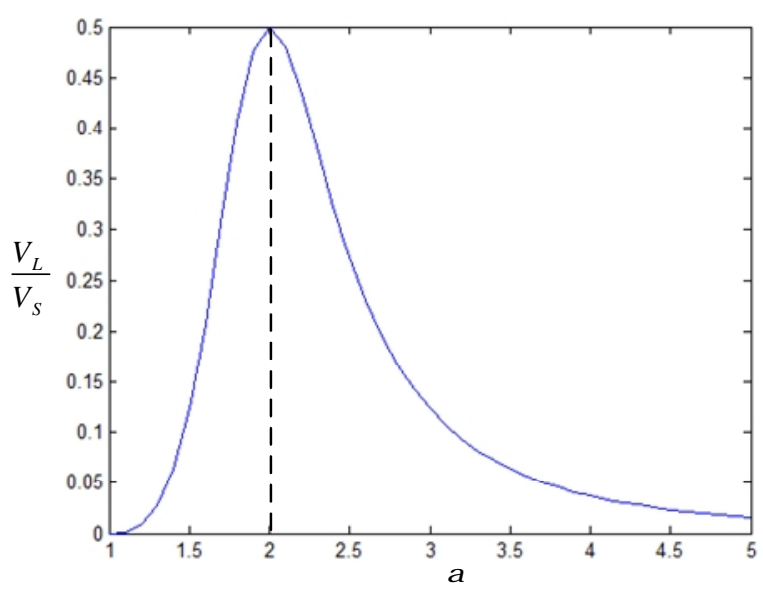

Fig. 3 The curves of load voltage for relay system

$$
\begin{aligned}
& \left(R_{1}+j \omega L+\frac{1}{j \omega C_{S}}\right) I_{S}+j \omega k L I_{R}=V_{S} \\
& \left(R_{1}+j \omega L+\frac{1}{j \omega C_{R}}\right) I_{R}+j \omega k L I_{S}=0 \\
& \frac{V_{L}}{V_{S}}=\frac{1}{k Q+\frac{1}{k Q}}
\end{aligned}
$$

Take advantage of the equivalent power between formal (3) and formal (15):

$$
\frac{1}{\frac{k_{1}}{k_{2}}+\frac{k_{2}}{k_{1}}}=\frac{1}{k Q+\frac{1}{k Q}}
$$

Take formal (10) and (11) into, it could get the relay coefficient mode:

$$
\begin{aligned}
& \alpha=(k Q)^{1 / 3}+1 \\
& \alpha=(k Q)^{-1 / 3}+1
\end{aligned}
$$

Because the reciprocity of system, so when $k Q>1$ in common WPT system, $(k Q)^{-1 / 3}+1 \leq \alpha \leq(k Q)^{1 / 3}+1$; when $k Q<1$ in common WPT system, $(k Q)^{1 / 3}+1 \leq \alpha \leq(k Q)^{-1 / 3}+1$; when $k Q=1$ in common WPT system, the common WPT system works at the maximum load voltage, the relay structure WPT system also works at the maximum load voltage, $\alpha=2 . k$ is the coupling coefficient of common WPT system, $Q$ is quality factor of common WPT system transmission terminal.

\section{Simulated analysis and experimental verification}

It has analyzed the power transfer processes through the theoretical model, this section will do simulation with relay structure WPT system by electromagnetic field simulation software. Use 
scattering parameter $S_{21}$ to express the system transfer efficiency. The vertical dimension between transmission terminal and receiving terminal is $60 \mathrm{~cm}$, it could get different transfer efficiency with the changing of position of relay structure shown as figure 4, what the figure 4(a) shown is magnetic field distribution of the relay structure WPT system when the relay structure located at the middle of the axis working at resonant condition, the magnetic field distribution could reflect the energy distribution in different construction, when the system was working at the resonant condition, the magnetic field distribution of system was symmetrical, it also express the system was working at the optimal operating condition when the energy distributed between transmission terminal and receiving terminal. What the figure 4(b) shown is the system transfer efficiency when the relay structure located at the middle of the axis, what the figure 4(c) shown is the system transfer efficiency when the relay structure located at the position deviate $5 \mathrm{~cm}$ away from the middle of the axis, what the figure 4(d) shown is the system transfer efficiency when the relay structure located at the position deviate $10 \mathrm{~cm}$ away from the middle of the axis. Because the system positive transfer coefficient has the relationship with load voltage ratio shown as formal (19)[9], and it also get that $R_{S}=R_{L}$ as the system impedance matching, so it could use $S_{21}$ to express the change rule of load voltage ratio.

$$
S_{21}=2 \frac{V_{L}}{V_{S}}\left(\frac{R_{S}}{R_{L}}\right)^{1 / 2}
$$

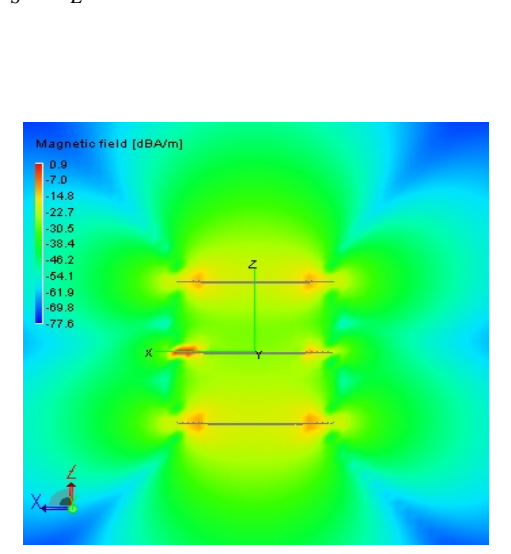

(a)

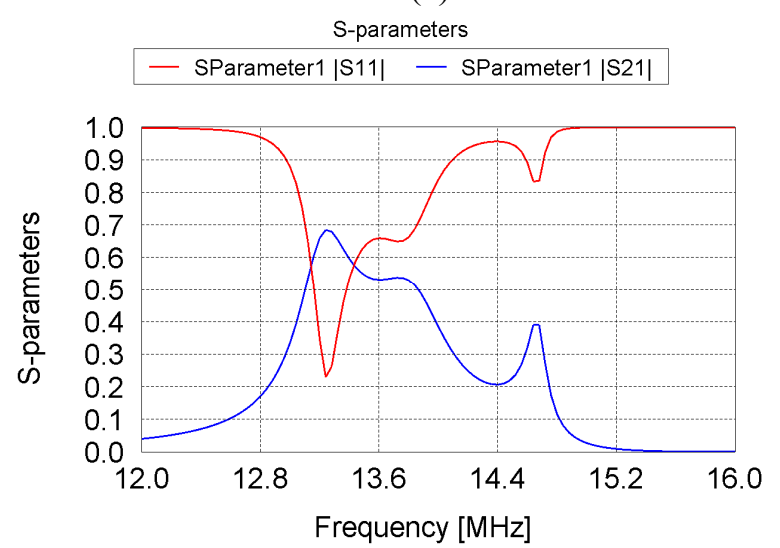

(c)

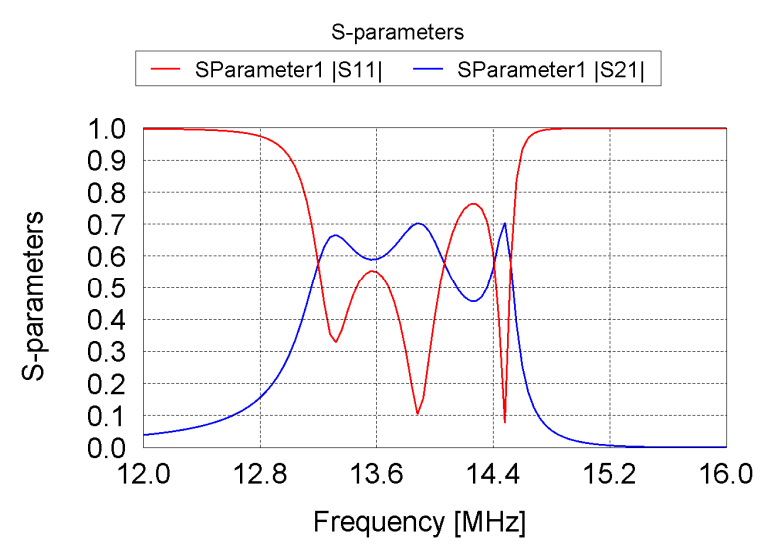

(b)

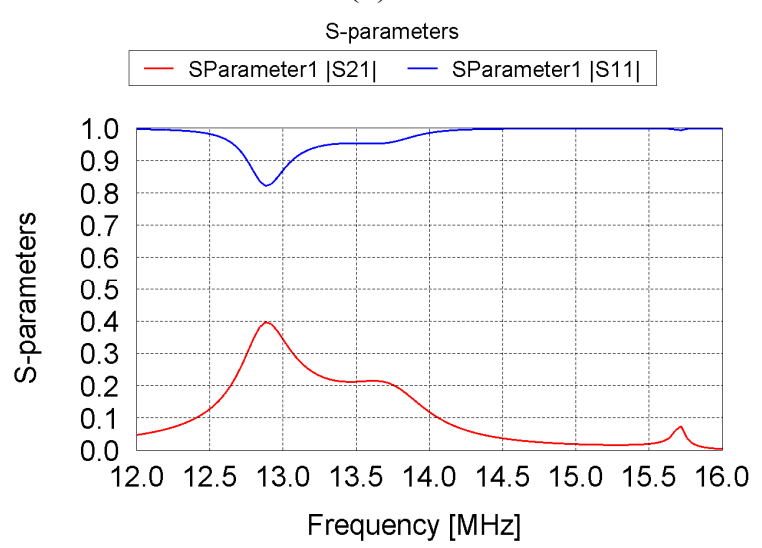

(d)

Fig.4 Relay system simulation

When the relay structure located at the middle of axis, the WPT system could maximum transfer efficiency and good frequency bandwidth, when the relay structure deviates away from the middle of the axis, the system transfer efficiency decline quickly. When the relay structure deviate $5 \mathrm{~cm}$, the system positive transfer coefficient is 0.51 , the load voltage ratio is 0.255 , the theoretical calculating value with mode is 0.265 ; When the relay structure deviate $10 \mathrm{~cm}$, the system positive transfer coefficient is 0.2 , the load voltage ratio is 0.1 , the theoretical calculating value with mode is 0.12 ; so the simulation value is correspondence with theoretical calculating value. 


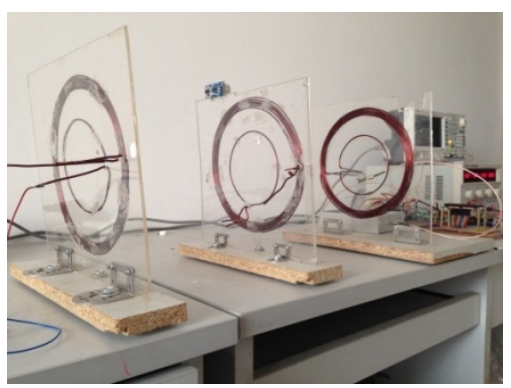

Fig.5 Relay system experiment

We exploit a WPT experimental platform, shown as figure 5, we make experimental verification for these three conditions.

In this experimental platform, high-frequency voltage is loaded at the exciting coil which was two stage amplified. We could light $4 \mathrm{~W}$ LED bulb at 0.8 meter away, and the luminance could be adjusted. In experiment, we use $50 \mathrm{ohm}$ resistance as the load. In the relay structure experiment, the transmission distance is $60 \mathrm{~cm}$, the inner radius of transmission terminal is $16.5 \mathrm{~cm}$, the extermal radius of transmission terminal is $19 \mathrm{~cm}$, number of turns is 6 , the coupling coefficient is 0.15 , the quality factor of system is 30 .

First, we verify the effect of relay structure to the transfer efficiency of WPT sytem. We measure the load voltage of relay structure WPT system with different relay structure location, and then we could calculate the load voltage ratio.

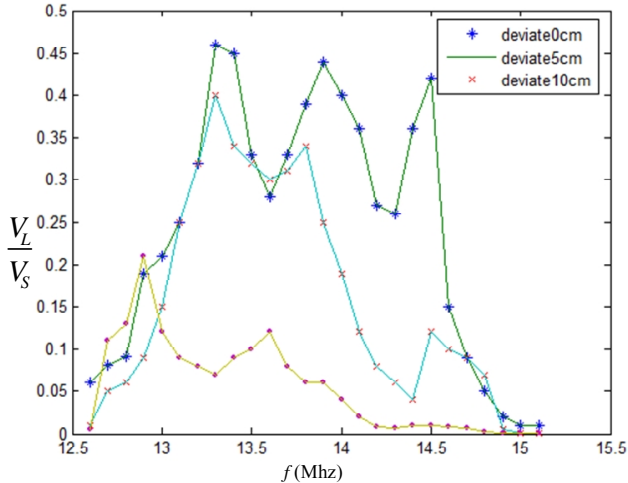

(a)

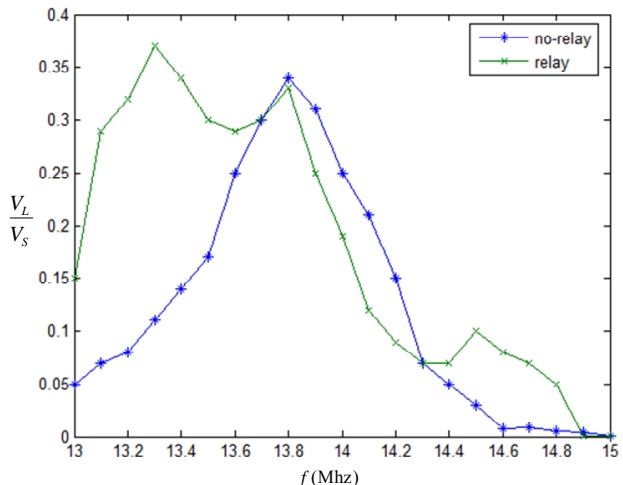

(b)

Fig.6 (a) Analysis of relay antenna position, (b) Verification of reasonable range for relay coefficient

Shown as figure 8 , we measure load voltage under three position condition respectively. the blue curve express the load voltage when the relay structure is at the middle of the axis; the green curve express the load voltage when the relay structure located at the position deviate $5 \mathrm{~cm}$ away from the middle of the axis; the red curve express the load voltage when the relay structure located at the position deviate $10 \mathrm{~cm}$ away from the middle of the axis. The curves of experimental measured have the same change rule with theoretical analysis and simulation analysis, it could prove when the relay structure at the middle of the axis, the system has the best working condition, when the relay structure located at the position deviate away from the middle of the axis, the transfer efficiency decline quickly. So the experiment proves the correctness of theoretical analysis.

The transfer efficiency comparison between the relay structure WPT system and the common WPT system is shown in figure 6(b). Because the relay structure could not always enhance the transfer efficiency of WPT system, if the relay structure was located too far away from the middle of axis, it could reduce the transfer efficiency quickly, so we can deduce there is range that for relay structure located to enhance the system transfer efficiency. We make an experiment to verify the range that calculated reasonable range for relay structure. We could calculate the range of relay coefficient as $1.6 \leq \alpha \leq 2.65$ through formal 17 , the relay structure located at the position deviate 7.5 $\mathrm{cm}$ away from the middle of the axis, compared with the common WPT system, the working frequency of the two system ara $13.81 \mathrm{Mhz}$, the load voltages of the two system respectively are 0.34 and 0.33 . In consideration of the system measuring error, we consider the two systems have the same transmission performance. The experimental result proved the correctness of relay coefficient 
and the reasonability of relay structure position range.

\section{Conclusion}

In this paper, we did a transmission performance research of relay structure Wireless Power Transmission System via Electromagnetic Resonant Coupling. First, we analyzed the energy exchange relations of system by coupled-mode theory, then, we analyzed the system transmission performance through circuit model. We got some conclusion as: (1) The relay structure WPT system has the same energy exchange relations with the common WPT system.(2) we deduced the effect of relay structure position for WPT system transmission performance, we also deduced the best position for relay structure, defined the notion of relay coefficient, then we got the mode of reasonable relay coefficient range. Finally, we make some experiment to verify our research.

\section{Reference}

[1] Andre Kurs, Aristeidis Karalis, Robert M offatt, et a1. W ireless power transfer via strongly coupled magnetic resonances[J]. Science, 2007, 317(6): 83-86.

[2] Yang Qingxin, Chen Haiyan, Xu Guizhi, et al. Research progress in contactless power transmission technology[J]. Transactions of China Electrotechnical Society, 2010, 25(7): 6-13.

[3] Li Yang, Yang Qingxin, Chen Haiyan, et a1. Influence factors analysis on power and efficiency in wireless power transfer system $[\mathrm{J}]$. Advanced Technology of Electrical Engineering and Energy, 2012, 31(3): 31-34, 39 .

[4] Huang Xueliang, Ji Qingjing, Tan Linlin, et al. Study on series-parallel model of wireless power transfer via magnetic resonance coupling[J]. Transactions of China Electrotechnical Society, 2013, 28(3): 171-176.

[5] Alanson P. Sample, David A. Meyer, and Joshua R.Smith. Analysis, Experimental Results, and Range Adaptation of Magnetically Coupled Resonators for Wireless Power Transfer[J]. IEEE Transactions on Industrial Electronics, 2011, 58(2):544-554.

[6] Hiroshi Hirayama, Toshiyuki Ozawa, Yosuke Hiraiwa, Nobuyoshi Kikuma, and Kunio Sakakibara. A Consideration of Electro-magnetic -resonant Coupling Mode in Wireless Power Transmission[J]. IEICE Electronics Express, 2009,6(19):1421-1425.

[7] Zhang Xian, Yang Qingxin, Zhang Xin, Li Yang, et al. Modeling and Experimental Verification for a Novel Wireless Power Transmission System via Electromagnetic Resonant Coupling[J]. Transactions of China Electrotechnical Society, 2013, 29(2): 185-190.

[8] Sanghoon Cheon, Yong-Hae Kim, Seung-Youl Kang, et al. Circuit-Model-Based Analysis of a Wireless Energy-Transfer System via Coupled Magnetic Resonances[J]. IEEE Transactions on Industrial Electronics, 2011, 58(7): 2906-2914.

[9] Linhui Chen, Shuo Liu, Yong Chun Zhou, and Tie Jun Cui. An Optimizable Circuit Structure for High Efficiency Wireless Power Transfer[J]. IEEE Transactions on Industrial Electronics, 2013, 60(1): 339-349. 